\title{
Analysis of Caesarean Sections According to Robson's Classification System in Rio De Janeiro
}

\author{
Lara Costa Kevorkian, Juliana Pontes Amaral, Marianna de Azevedo de Yparraguirre Spínola, Vander Guimarães \\ Silva, Julia Barban Morelli Rosas and Regis Rodrigues Vieira*
}

Faculdade de Medicina de Petrópolis, Brazil

Submission: December 12, 2018 ; Published: December 21, 2018

*Corresponding author: Regis Rodrigues Vieira, Faculdade de Medicina de Petrópolis, Brazil

\begin{abstract}
Since 2015 the World Health Organization recommended that the Robson's Ten Group Classification System (TGCS) should be instituted in all healthcare facilities in the delivery field. The TGCS is a robust method, with great analytical power and simple implementation. It can provide an overview of an institution's obstetric profile, making it possible to analyze the quality of the service, which results in assisting improvements. It also generates information that allows the conception of public policies in order to reduce CS rates. This study's objective was to use the TGCS to outline for the first time the cesarean profile of the Petrópolis Teaching Hospital (PTH). This is a retrospective, quantitative, cross-sectional study, based on the analysis of medical records of 1180 deliveries at PTH in 2016 (40\% of total). The obtained data was tabulated in Windows Excel spreadsheets and compared with data from Ireland and general Brazil studies. The groups with the biggest relative sizes were $2(20,48 \%), 5(20,22 \%), 10(16,11 \%)$, which were also the ones with the highest relative contribution rates on the overall CS rate (respectively, 23.58\%, 32.42\%, 20.63\%). In Ireland, the relative contribution of the same groups is $23.83 \%, 28.97 \%, 6.54 \%$, whereas in Brazil it's $28.6 \%, 31.1 \%, 9.8 \%$. The high contribution of group 2 is responsible to increase group 5, since multiparous with previous CS often leads to a new CS. To reduce these rates, new policies should be created and a complete adhesion of induction protocols, installed. The discrepancy of group 10 happens because PTH's the only local high risk maternity hospital, however premature CS indication should be carefully addressed.
\end{abstract}

Keywords: Evidence-based medicine; Robson Classification; Caesarean section

Abbreviatations: WHO: World Health Organization; TGCS: Ten Group Classification System; CS: Caesarean Section; PTH: Petrópolis Teaching Hospital

\section{Introduction}

Since 2016, FIGO has stated, reaffirming the 2015 the World Health Organization (WHO) recommendation, that the Robson's Ten Group Classification System (TGCS) should be instituted in all healthcare facilities in the maternity and delivery field. Both recommendations were a result of a previous search for a method that could analyze all elements directly involved in the crescent numbers of caesarean section (CS) rates throughout the world, comparing them in a more suitable way. According to WHO, the use of a reliable and internationally accepted classification system to produce standardized data enables comparisons across populations, providing tools to investigate drivers of the upward trend in caesarean section $[1,2]$.

In this context, the classification system developed by the Irish obstetrician Michael Robson was a perfect fit to reach the goals laid out by WHO, producing reliable data that allows the comparison of different countries, cities, and health institutions. The TGCS is, also, robust method, with great analytical power, low cost and simple implementation. To Clode [3], Portuguese obstetrician, "the simplicity of concepts, easy interpretation and comprehension of objectives were aspects that contributed to the rapid adhesion to the TGCS". The TGCS uses obstetric parameters to allocate all the pregnant women that arrive in a maternity hospital in one of the proposed 10 groups. These groups were structured according to four main criteria: previous obstetric record (parity and existence, or not, of a previous CS), pregnancy category (foetal presentation and number of foetuses), onset of labor (spontaneous, induced or pre-labor CS) and gestational age at the moment of delivery [4].

Amongst the advantages of using the TGCS is the fact that it can also provide an overview of an institution's obstetric profile, something that can be periodically made, offering a longitudinal study of the patterns found in a certain maternity hospital and its changes over time. This process makes it possible to evaluate the quality of the service, which can lead to assisting improvements. This can be extremely useful when it occurs in a local referral healthcare service, which has great demands from the users and great delivery numbers $[3,5]$. Over the last decade, it was possible 
to observe a worrisome scenario concerning perinatal assistance in Brazil, in which CS rates came close to 60\% [6]. In 2015 WHO pointed out that the country is the world's leader in CS numbers and warned about a possible outbreak of surgical deliveries. In this context, the TGCS could be a key instrument not only to evaluate a healthcare institution and compare it with other institutions, but also to promote changes focusing on diminishing CS rates, by optimizing the use of these surgeries. This could be made focusing interventions on specific groups of particular relevance for each maternity hospital $[2,7]$.

Provided that, the use of the TGCS in Petrópolis Teaching Hospital (PTH) is relevant, considering it is the main high risk obstetric service of the region where it is located and the only public maternity hospital in the city of Petrópolis, Rio de Janeiro state, Brazil. Annually, the PTH performs 4000 obstetric consultations and 2800 deliveries [8].The main objective of this paper, thus, was to unravel the obstetric profile of PTH and deepen the knowledge about the CS rates patterns that occurs in this high risk maternity hospital. Furthermore, the use of the TGCS is particularly significant, considering that Brazil has extremely high CS rates, coming close to $60 \%$ of all deliveries in the public health care unified system [6]. Given this, this paper also aims to spread and deepen the TGCS use in a more local scale, in order to effectively reduce CS rates in the country [9].

\section{Materials and Methods}

This is a retrospective, quantitative, cross-sectional study that has analytical character. It is based on the analysis of medical records of 1180 women who gave birth in 2016 (40\% of total deliveries), at PTH, the only local high risk maternity hospital, located in Rio de Janeiro state, Brazil. The data collection was made according to the TGCS, using a questionnaire that also included the mother's age and the delivery outcome. Were included in the analysis the medical records that were available in the PTH's archive, that contained: parity, gestational age, number of foetuses (one or multiple), foetal presentation, onset of labor (spontaneous, induced or pre-labor CS), delivery type (vaginal or $\mathrm{CS}$ and the previous existence, or not, of a CS). The medical records that were incorrectly or incompletely filled were excluded. As the development of this study involved the use of patient records, its research protocol was approved by the Ethics Committee before the beginning of data collection. An informed consent form was not needed, considering it is a retrospective and analytical study and, therefore, did not make any kind of interventions nor needed to directly collect information from its subjects.

The obtained data was tabulated in Windows excel spreadsheets 2010, using it to calculate each group size and its contributions to the overall CS rates. This information was also compared to the data from the "Birth in Brazil Survey", a Brazilian national project, and the original Robson's works, from Ireland. After the completion of the analysis, the numbers were exposed to the hospital's gynecology and obstetrics department of PTH/Petrópolis Medical School.

\section{Result}

A total of 1180 records were randomly selected, of women whose births took place in 2016. Thirteen participants were excluded due to incomplete or incorrect filling. Thus, 1167 medical records were analyzed, of which 692 represented vaginal deliveries and 475 caesarean sections, resulting in the PTH's caesarean rate of $40.7 \%$. Group 2 has the biggest relative size, followed by groups 5, 10 and 1, which represents PTH's obstetric profile. All women in group 9 (single pregnancy with transverse or oblique lie) underwent CS. The highest CS rates in each group, following group 9, were found in groups 7, 8 and 5. The lowest CS rate was found in group 3. However, this scenario somewhat changes when it comes to the contribution of each group to the overall CS rate, with the main contributor being group 5 (women with previous CS), followed by groups 2, 10 and 1. Opposite to the aforementioned data, group 9 has the lowest contribution (less than $1 \%)$.

\section{Discussion}

PTH is the learning scenario of the medicine and nursing graduation and post-graduation students from Petrópolis Medical School/Arthur Sá Earp Neto School. Thus, the teaching happening there should be based on the quality of practices, valuing the scientific innovations and the evidence-based medicine, according to the tendencies that have been establishing themselves in the international academic community [10]. Considering this, a feedback to the maternity teaching hospital consisting of the data collected and its analysis can be used as a source of information based on which changes can be made possible. Furthermore, the outcomes in each group are capable of providing a better understanding of the healthcare institution's obstetric profile $[2,11,12]$.

The obstetric profile found in PTH is the same found in the "Birth in Brazil Survey", representing Brazil's reality in the public health care unified system, and in Robson's works, representing the scenario existent in Ireland, both studies having groups 2, 5 and 10 as the groups with biggest relative sizes. These groups also had the highest relative contributions to the overall CS rates in PTH: 2 (23.58\%), 5 (32.42\%), 10 (20.63\%). In Ireland the relative contribution of the same groups on the total CS rate is, respectively: $23.83 \%, 28.97 \%, 6.54 \%$, whereas in Brazil they are, $28.6 \%, 31.1 \%, 9.8 \%$. The CS rates found in PTH, Brazil and Ireland were, respectively, $40.7 \%, 42.2 \%$ and $23.1 \%$ [4,7].

Group 2 is composed by nulliparous with single cephalic pregnancy, >37 weeks gestation who either had labor induced or were delivered by CS before labor. Its high contribution to the overall CS rates in PTH, following the pattern also found in the "Birth in Brazil Survey", leads to an increase of the number of multiparous with previous CS. Therefore, in a next pregnancy, these women are going to be allocated in group 5 and are more likely to have a new CS, considering the established culture of indicating the surgery to all the women who already have a uterine scar. Group 5, therefore, becomes the highest CS rate contributor, 
on account of the aforementioned domino effect that also was found in PTH, "Birth in Brazil Survey" and in Ireland $[7,13]$.

In PTH, group 10 (all women with a single cephalic pregnancy, $<37$ weeks gestation, including women with previous uterine scars) had a higher contribution to the overall CS rates when compared to "Birth in Brazil Survey" and Ireland. Taking into account that the group is composed of premature deliveries, it is one of the main groups in which high risk obstetrical interventions often need to be performed, requiring specialized assistance. Such facts can partially explain the discrepancy that was found, PTH being the only local high risk maternity hospital. Nonetheless, premature CS indications should be more carefully addressed.

Although the biggest CS rates were found in groups 9, 10 and 8 , in absolute numbers, these groups are not responsible for the biggest relative contributions to the overall CS rate, as seen previously. This observation has extreme relevance when it comes to planning new intern policies in assistance, given that groups 2 , 5 and 10 should be the focus of those actions. Therefore, the target of the improvements of a healthcare service should be based on the groups with biggest relative contribution to the overall CS rate, and the absolute number of each group should be analyzed individually, taking into account the context in which the hospital is set in [11].

These improvements can only be made after a careful analysis of the data collected, transforming numbers into useful information in which the changes should be based on [14]. In PTH, the results found after the completion of the analysis of this paper were exposed to the hospital's gynaecology and obstetrics department. It was observed that the TGCS is still a pretty recent device, whose knowledge hasn't spread itself deeply into all maternity hospitals. Thus, it is not comprehended to its full depth by the healthcare professionals in a more local scale, despite the 2015 WHO statement on CS rates and FIGO's 2016 reinforcement on that matter [1,2]. Apart from that, it was observed a great number of incomplete or wrongly filled medical records, resulting in 13 of them that could not be classified. This number is surprising, given that in the "Birth in Brazil Survey", which included 23,894 women, only seven were not able to be classified. This information suggests that the training in filling such documents is not being correctly applied, a fact that was also addressed in the feedback stage of the project [7].

In this scenario, the use of the TGCS provides information that allows the conception of public policies in order to reduce CS rates. Despite the locally found unfamiliarity of healthcare professionals with the TGCS, it has been employed in a crescent way, more recently [15]. Brazil's Ministry of Health, alongside national research institutions, such as the Oswaldo Cruz Foundation/ National Institute of Women, Children and Adolescents Health Fernandes Figueira, are engaging in transform the high CS rates scenario in Brazil using the TGCS. One example of this process is the creation of the "Apice on" project, a program of enhancement and innovation in assistance and teaching of obstetrics and neonatology. Another important step was the availability of the TGCS national data in the public healthcare unified system online database, DATASUS $[16,17]$.

In order to solve the domino effect involving groups 2 and 5 seen in PTH and in Brazil, as shown in "Birth in Brazil Survey", it is indicated to prevent the first caesarean by implementing a more complete adhesion of induction protocols, using it to its full extent with appropriate management, instead of immediately set sail for a CS before labor. In addition, encouraging vaginal birth after CS (VBAC) and deconstructing the stigma of "once a caesarean, always a caesarean" is a fundamental step to effectively reduce CS rates $[7,18]$.

\section{Conclusion}

Considering the aforementioned, in order to solve the domino effect involving groups 2 and 5 seen in PTH and in Brazil, as shown in "Birth in Brazil Survey", it is indicated to prevent the first caesarean by implementing a more complete adhesion of induction protocols, using it to its full extent with appropriate management, instead of immediately set sail for a CS before labor. Encouraging vaginal birth after CS (VBAC) and deconstructing the stigma of "once a caesarean, always a caesarean" is also a fundamental step to effectively reduce CS rates. In addition, it is extremely relevant to spread and establish the use of the TGCS in all healthcare facilities, as well as encourage medical and nursing teaching institutions to adhere to programs that value innovative practices seeking changes in Brazil's obstetric profile $[7,16,18]$.

\section{References}

1. FIGO Working Group on Challenges in Care of Mothers and Infants during Labor and Delivery (2016) Best practice advice on the 10-Group Classification System for caesarean deliveries. Int J Gynecol Obstet 135(2): 232-233.

2. World Health Organization (2015) WHO Statement on Caesarean Section Rates. Geneva.

3. Clode N (2017) The ten group classification system (Robson Classification): just a caesarean classification? Acta Obstet Ginecol Port 11(2): 80-82.

4. Robson M, Hartigan L, Murphy M (2013) Methods of achieving and maintaining an appropriate caesarean section rate. Best Pract Res Clin Obstet Gynaecol 27(2): 297-308.

5. Robson MS (2001) Classification of caesarean sections. Fetal Matern Med Rev 12(1): 23-39.

6. Ministry of Health (BR) (2016) MS/SVS/DASIS: Live Birth Information System. Live births in 2016 by type of delivery. Brasília: Ministry of Health.

7. Nakamura-Pereira M, Leal MC, Esteves-Pereira AP, Domingues RMSM, Torres JA, et al. (2016) Use of Robson classification to assess caesarean section rate in Brazil: the role of source of payment for childbirth. Reprod Health 13(Suppl 3): 245-256.

8. Alcides Carneiro Hospital (2016) System of Management Information. Petrópolis: Sehac.

9. Betrán AP, Vindevoghel N, Souza JP, Gulmezoglu AM, Torloni MR, et al. (2014) A systematic review of the Robson Classification for Caesarean Section: What Works, Doesn't Work and How to Improve It. PLoS One 9(6): e97769. 
10. Macha S, Muyuni M, Nkonde S, Faúndes A (2014) Increasing access to legal termination of pregnancy and post abortion contraception at the University Teaching Hospital, Lusaka, Zambia. Int J Gynecol Obstet, 126(1): S49-S51.

11. Robson M, Murphy M, Byrne F (2015) Quality assurance: The 10-Group Classification System (Robson classification), induction of labor, and caesarean delivery. Int J Gynecol Obstet 131(Suppl 1): S23-27.

12. Betrán AP, Gulmezoglu AM, Robson M, Merialdi M, Souza JP, et al (2009) WHO Global Survey on Maternal and Perinatal Health in Latin America: classifying caesarean sections. Reprod Health 6: 18.

13. Vogel JP, Betrán AP, Vindevoghel N, Souza JP, Torloni MR, et al. (2015) Use of the Robson classification to assess caesarean section trends in 21 countries: a secondary analysis of two WHO multicountry surveys. Lancet Glob Health 3(5): e260-270.

14. Ferreira EC, Pacagnella RC, Costa ML, Cecatti JG (2015) The Robson

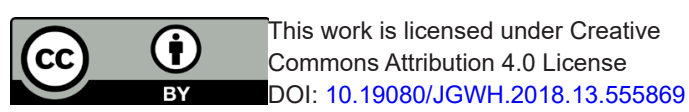

ten-group classification system for appraising deliveries at a tertiary referral hospital in Brazil. Int J Gynecol Obstet 129(3): 236-239.

15. Boatin AA, Cullinane F, Torloni MR, Betrán AP (2017) Audit and feedback using the Robson classification to reduce caesarean section rates: a systematic review. BJOG 25(1): 36-42.

16. Ministry of Health (BR) (2017) Apice On-Improvement and Innovation Project in Care and Teaching in Obstetrics and Neonatology. Brasília: Ministry of Health.

17. Ministry of Health (BR) (2016) MS/SVS/DASIS: Live Birth Information System. Live births in 2016 by type of delivery. Brasília: Ministry of Health.

18. Triunfo S, Ferrazzani S, Lanzone A, Scambia G (2015) Identification of obstetric targets for reducing caesarean section rate using the Robson Ten Group Classification in a tertiary level hospital. Eur J Obstet Gynecol Reprod Biol 189: 91-95.

Your next submission with Juniper Publishers will reach you the below assets

- Quality Editorial service

- Swift Peer Review

- Reprints availability

- E-prints Service

- Manuscript Podcast for convenient understanding

- Global attainment for your research

- Manuscript accessibility in different formats ( Pdf, E-pub, Full Text, Audio)

- Unceasing customer service

Track the below URL for one-step submission https://juniperpublishers.com/online-submission.php 